\title{
OPEN Olfactory function testing before and after anesthesia
}

\author{
Anna Kristina Hernandez ${ }^{\bowtie}$, Patrick Fuchss, Antje Haehner \& Thomas Hummel
}

This study aimed to determine whether anesthesia would affect olfactory function. Patients who were admitted for surgical intervention that did not include the nasal cavity and paranasal sinuses were included in this prospective cohort study. Structured medical history was taken from the patients, including the following: age, sex, smoking history, alcohol intake, current medications, and sleep deficits prior to surgery. Before surgery, patients were asked for a self-rating of their olfactory function. Olfactory function was also measured using Sniffin' Sticks comprising measures of odor threshold, discrimination, and identification. The mean interval between olfactory tests was 6 days (range 3-12 days). Seventy-three patients were included in the study, 34 men and 39 women. Olfactory scores were consistent before and after surgery as indicated by correlative analyses $(p<0.05)$. Odor thresholds, discrimination, identification, and composite TDI scores did not change significantly, whereas odor identification scores increased $(p=0.011)$ after surgery. In conclusion, postoperative olfactory scores remained stable. However, identification scores exhibited a slight increase which can be attributed to a retest effect. Overall, the present results indicate that surgery outside of the nasal and paranasal sinus region performed in general anesthesia has no major effect on the sense of smell.

Olfactory dysfunction occurs in approximately $5 \%$ of the general population ${ }^{1,2}$. Commonly reported etiologies include sinonasal conditions, viral infection, craniofacial trauma, neurodegenerative disorders, and drugs ${ }^{2-6}$. Postoperative changes to olfactory function are often observed following sinonasal ${ }^{7-12}$ and neurosurgery ${ }^{13-17}$, especially when changes occur in areas related to olfaction such as the olfactory bulb, cribriform plate, olfactory mucosa, or nasal septum. In the last 30 years, however, postoperative changes to olfaction have been also reported in other types of surgery, such as: cardiovascular ${ }^{18,19}$, abdominal ${ }^{20-23}$, and genito-urinary ${ }^{24-26}$.

There have also been several reports on effects of anesthesia ${ }^{27}$ on olfaction. Most reports involve general anesthesia ${ }^{21-24,26,28-30}$, however, there are a few citing spinal anesthesia ${ }^{3,31}$, intravenous ${ }^{25}$, and topical anesthesia ${ }^{28,32,33}$ to also have effects on olfaction. The rarity of such events may also mean that these cases are underreported.

The primary aim of the study was to determine the effect of anesthesia on olfactory function, among patients who underwent non-nasal surgery. To this end, patients should not only rate their sense of smell but olfactory function was measured in great detail to allow for relatively unbiased detection of possible subtle changes in olfactory sensitivity. Secondarily, we aimed to determine the relationship of the following variables: age, sex, smoking, alcohol intake, concomitant medication, pre-operative self-rating of olfactory function, preoperative sleep deficit, type of surgery, and duration of surgery to olfactory function score change after surgery among patients undergoing non-nasal surgeries.

\section{Materials and methods}

This study was approved by the Institutional Review Board (IRB) of TU Dresden and was conducted according to the principles expressed in the Declaration of Helsinki. The study included a total of 73 patients (34 men and 39 women) of at least 18 years of age. Patients were admitted for surgical intervention at the Department of Orthopedics, Trauma and Reconstructive Surgery or the Department of Otorhinolaryngology at the University Clinic in Dresden. Inclusion and exclusion criteria can be found in Table 1.

Following a patient's admission to the hospital, an explanation describing all possible risks and benefits related to participation in the study was given. Patients who gave their written informed consent to participate were interviewed. A structured medical history was taken ${ }^{34}$. This included the following details: age, sex, smoking history, alcohol intake, current medications, sleep deficits prior to surgery, and pre-operative olfactory self-rating. 


\begin{tabular}{|l|l|}
\hline Inclusion criteria & Exclusion criteria \\
\hline At least 18 years old & Relevant previous ENT diseases (i.e., Chronic Rhinosinusitis, Allergic Rhinitis, Nasal Polyps) \\
\hline Hospitalization for surgical treatment & Acute or pronounced chronic sinonasal inflammation \\
\hline At least 3 days of hospitalization & Significant health impairment associated with olfactory disorders \\
\hline
\end{tabular}

Table 1. Inclusion and exclusion criteria.

Olfactory testing was done using the Sniffin' Sticks test battery (Burghart Messtechnik, Wedel, Germany) ${ }^{35}$. Using commercially available felt-tip pens, a combination of olfactory threshold, odor discrimination, and odor identification score was determined.

For olfactory thresholds, a three-alternative forced-choice method was used. Three pens were held in alternating order at a distance of about two centimeters in front of both nostrils. Two of these pens contained an odorless solvent and one pen contained phenylethyl alcohol (PEA), a rose-like odor. The test contains 16 sets of three pens, each set with an increasing concentration of PEA. Set 16 has the lowest concentration of PEA, while set one has the highest. Participants were asked to select which pen among each triplet contained the odorant. Starting with the lowest odor concentration, a stepwise testing was done where two consecutive correct identifications of the odorous pen or one incorrect answer marked a "turning point". This resulted to the presentation of the next triplet in decreasing or increasing concentration, respectively. The triplets were presented 20 to $30 \mathrm{~s}$ apart. The threshold score was defined as the average value of the last four turning points, with the final score ranging from 1 to 16 . The participants were blindfolded to avoid visual identification of target pens. For odor discrimination, the same three-alternative forced choice method was used. Here, each set of three pens contained two pens with the same odorant and one pen with a different odorant. The participants were asked to identify the pen with the different odorant, in each of the 16 triplets. The score was the total number of correctly identified odorant pens, with a range of 0 to 16 points. Similar to the threshold subtest, participants were also blindfolded during the discrimination subtest to avoid visual identification of target pens. For odor identification, each participant was presented with 16 pens containing common and familiar odorants. Each participant must correctly identify and label each odorant using a list of four alternative descriptors. The descriptors were presented to the participants as labeled pictures. The interval between presentation of each pen was 20-30 s. The identification score was the sum of correctly identified odorant pens, and scores may range between 0 to 16 points. The composite TDI Score was the sum of scores for threshold, discrimination, and identification subtests, with a range of 1 to 48 points.

Participants were prohibited from eating, drinking anything other than water, and smoking one hour before the olfactory test. The first test was done the night before the surgery or on the morning before the scheduled surgery. Tests were usually done in the patients' rooms, with careful consideration and measures to make the space as ideal as possible for testing (minimize other sources of odor/distractions or change study site as necessary). Shortly before patients were discharged, a second olfactory test was done under conditions comparable to the first test. In the case of multi-bed rooms, oral informed consent was obtained from all the other patients in the room prior to testing.

Patient records were assigned codes and anonymized. Data were encoded into a Microsoft Excel Office 365 version 2107 database (Microsoft Corp., Redmond, WA, USA) and checked for accuracy of encoding. Data analysis was done using SPSS ver. 28.0 (IBM, Chicago, IL, USA). Means and proportions were used to describe the study variables.

Pearson's $r$ was computed for age; Spearman's rho for smoking, alcohol intake, pre-operative olfactory selfrating; independent two-tailed t-tests for sex and sleep deficits; paired two-tailed t-tests for pre- and postoperative TDI scores; repeated measures ANOVA for types of surgery and medications in relation to TDI scores, post-operative change in TDI scores; and with a $p$ value of $<0.05$ considered significant.

\section{Results}

A total of 34 men and 39 women participated in the study, with ages ranging from 18 to 80 years old and a mean age of 51 years. The average olfactory testing interval was 6 days (SD 2.1, range 3-12 days). Most of the patients were non-smokers $(n=40,55 \%)$, with occasional alcohol intake $(n=52,71 \%)$, and without any current medications $(n=38,52 \%)$. The majority of the individuals rated themselves to have normal olfaction $(n=48,66 \%)$ and reported to have no sleep deficits prior to surgery $(n=48,66 \%)$.

The types of surgeries the patients underwent are listed on Table 2. Most common surgeries done were hip endoprostheses $(n=17,23 \%)$ and general hip surgery $(n=13,18 \%)$. The mean duration of surgery was $76 \mathrm{~min}$ (SD 22.1, range 30 to $180 \mathrm{~min}$ ) and the most common type of anesthesia used was general anesthesia $(\mathrm{n}=62$, $85 \%)$. Most people reported no change in olfactory self-ratings after surgery $(n=64,88 \%)$.

Pre- and post-operative change in composite TDI scores. Most post-operative changes to scores were observed in the threshold subtest, with less changes in the discrimination and identification subtests. Nine patients showed a change of at least 5.5 points on their post-operative TDI scores which is regarded as clinically significant ${ }^{36}$, four had worse TDI scores ("worse" group), while five had improved TDI scores ("better" group) (Table 3). All of the patients who experienced composite TDI score changes received general anesthesia (worse: $\mathrm{n}=4$, better: $\mathrm{n}=5$ ). 


\begin{tabular}{|c|c|c|}
\hline Variables & Frequency & Mean (SD) \\
\hline Age (in years) & & $51.01(17.44)$ \\
\hline \multicolumn{3}{|l|}{ Sex } \\
\hline Male & $34(46.6 \%)$ & \\
\hline Female & $39(53.4 \%)$ & \\
\hline \multicolumn{3}{|l|}{ Smoking } \\
\hline Never smoked & $40(54.8 \%)$ & \\
\hline Stopped for > lyear & $16(21.9 \%)$ & \\
\hline Smoker & $17(23.3 \%)$ & \\
\hline \multicolumn{3}{|l|}{ Alcohol intake } \\
\hline Never & $17(23.3 \%)$ & \\
\hline Occasionally & $52(71.2 \%)$ & \\
\hline Regularly & $4(5.5 \%)$ & \\
\hline \multicolumn{3}{|l|}{ Medications } \\
\hline No concomitant medication & $38(52.1 \%)$ & \\
\hline Anti-hypertension & $19(26.0 \%)$ & \\
\hline Nonsteroidal Anti-inflammatory Drugs (NSAIDs) & $5(6.8 \%)$ & \\
\hline Oral Contraceptives & $4(5.5 \%)$ & \\
\hline NSAIDs and Anti-hypertension & $6(8.2 \%)$ & \\
\hline Others & $1(1.4 \%)$ & \\
\hline \multicolumn{3}{|l|}{ Pre-operative olfactory self-rating } \\
\hline Worse & $9(12.3 \%)$ & \\
\hline Normal & $48(65.8 \%)$ & \\
\hline Better & $16(21.9 \%)$ & \\
\hline \multicolumn{3}{|l|}{ Sleep deficit } \\
\hline No & $48(65.8 \%)$ & \\
\hline Yes & $25(34.2 \%)$ & \\
\hline \multicolumn{3}{|l|}{ Operation } \\
\hline Foot & $10(13.7 \%)$ & \\
\hline Hip & $13(17.8 \%)$ & \\
\hline Knee & $4(5.5 \%)$ & \\
\hline Spine & $6(8.2 \%)$ & \\
\hline Upper Extremity & $5(6.8 \%)$ & \\
\hline Hip Endoprosthesis & $17(23.3 \%)$ & \\
\hline Knee Endoprosthesis & $8(11 \%)$ & \\
\hline Ear & $9(12.3 \%)$ & \\
\hline Throat & $1(1.4 \%)$ & \\
\hline Duration of surgery (in minutes) & & $76.8(22.1)$ \\
\hline \multicolumn{3}{|l|}{ Type of anesthesia } \\
\hline General & $62(84.9 \%)$ & \\
\hline Spinal & $1(1.4 \%)$ & \\
\hline Nerve block & $10(13.7 \%)$ & \\
\hline Olfactory testing interval (in days) & & $6.1(2.1)$ \\
\hline
\end{tabular}

Table 2. Frequencies, percentages and means.

\begin{tabular}{|l|l|l|l|l|}
\hline & Threshold $^{\mathbf{a}}$ & Discrimination $^{\mathbf{b}}$ & Identification $^{\mathbf{b}}$ & ${\text { Composite } \text { TDI }^{\mathbf{c}}}^{\mathbf{2}}$ \\
\hline Worse & $11(15.1 \%)$ & $9(12.2 \%)$ & $1(1.4 \%)$ & $4(5.5 \%)$ \\
\hline Same & $49(67.1 \%)$ & $59(79.7 \%)$ & $66(90.4 \%)$ & $64(87.7 \%)$ \\
\hline Better & $13(17.8 \%)$ & $5(6.8 \%)$ & $6(8.2 \%)$ & $5(6.9 \%)$ \\
\hline Total & $24(32.9 \%)$ & $14(19 \%)$ & $7(9.6 \%)$ & $9(12.4 \%)$ \\
\hline
\end{tabular}

Table 3. Summary of changes in threshold, discrimination, identification, and composite TDI scores. Scores were classified as "worse" or "better" when changes were greater or equal to the following: a2.5 points, b3 points, ${ }^{c} 5.5$ points ${ }^{36}$. 


\section{Composite TDI Score}

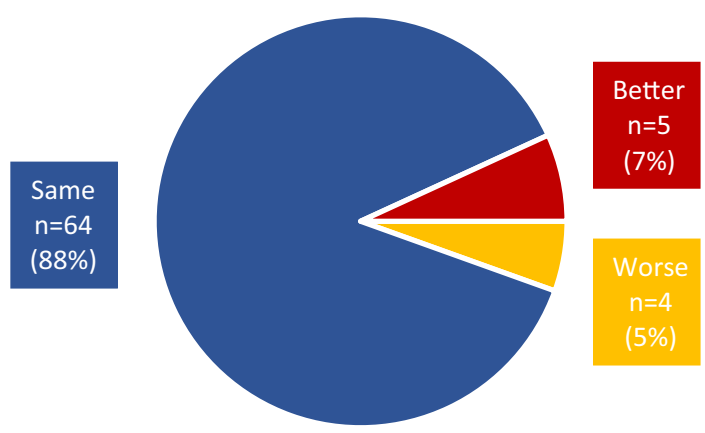

Figure 1. Pie chart of clinically significant changes in post-operative TDI Scores.

Differences between patients improving and those worsening in function. Mean age of those who had improved TDI scores was lower (age $=47$ years) versus those whose scores worsened (age $=65$ years). More women $(n=6)$ noted changes in their TDI scores than men $(n=3)$. However, only women $(n=4)$ had worse TDI scores, while men $(n=3)$ only had improved TDI scores (Fig. 1). The interval between testing was roughly the same between groups (range $=5-7$ days). Hip endoprosthesis surgery had the most number of patients with changes in TDI score (worse: $n=2$; better: $n=3$ ). The worse and better groups were roughly the same in terms of smoking, alcohol intake, concurrent medication, and sleep deficit. Most of the patients that reported changes to TDI score were non-smokers $(n=8)$ with occasional alcohol intake $(n=7)$, and no concurrent medications $(n=6)$. Most individuals with changes in TDI score had no sleep deficits $(n=8)$. However, the group with changes to threshold scores had a larger number of participants with sleep deficits (worse: $n=4$; better: $n=3$ ). Mean duration of surgery was longer for patients with worse TDI score (duration $=88 \mathrm{~min}$ ) versus those whose scores got better (duration $=70 \mathrm{~min}$ ).

Patients had higher identification scores after surgery $\left(t_{72}=2.60, p=0.011\right)$, with the mean post-operative identification score being 0.47 points higher than the mean of pre-operative scores (95\% CI [0.109, 0.822]). Threshold, discrimination, and composite TDI scores had no significant difference before and after surgery.

Secondary questions. Age was negatively correlated with changes in threshold score, but not discrimination, identification or composite TDI score. This means, the younger an individual, the more likely for threshold scores to improve post-operatively $\left(\mathrm{rs}_{71}=-0.28, p=0.018\right)$.

Sleep deficit had a significant effect on threshold scores, $\mathrm{F}_{1,71}=8.953, p=0.004$. Patients without sleep deficits had significantly higher threshold scores pre- $\left(\mathrm{t}_{72}=2.63, p=0.011\right)$ and post-operatively $\left(\mathrm{t}_{72}=2.57, p=0.012\right)$.

Age was negatively correlated with pre-operative discrimination $\left(\mathrm{r}_{71}=-0.46, p<0.001\right)$, pre-operative identification $\left(\mathrm{r}_{71}=-0.38, p<0.001\right)$, and pre-operative composite TDI score $\left(\mathrm{r}_{71}=-0.46, p<0.001\right)$, but not preoperative threshold score $\left(\mathrm{r}_{71}=-0.19, p=0.115\right)$.

Olfactory testing interval, duration of surgery, smoking, alcohol intake, and pre-operative self-rating of olfactory function were not correlated with changes in odor threshold, discrimination, identification, and composite TDI scores. Type of surgery or current medication had no effect on TDI scores.

\section{Discussion}

There are only a few published articles about changes in olfaction related to anesthesia, and most of these are case reports or letters to editors. Some have attempted to determine the effect of anesthesia in controlled studies, however, only transient effects were noted at best $\mathrm{t}^{30,33}$. There has been no clear causality or pathophysiology for these phenomena. Despite the presence of significant improvement in the identification scores among postoperative patients in this study, administration of anesthesia during surgery did not have a significant association with this change. We hypothesize that this observed change may simply be due to retest effect, which is defined as the increase in test scores due to the repeated administration of cognitive ability tests ${ }^{37,38}$. As odor identification testing is known to be affected by cognitive function, particularly executive processes and semantic memory $\mathrm{y}^{1,35,39}$, we believe that retest effect may also apply to odor identification testing. An increase in odor identification scores with repeated testing has been previously reported in literature ${ }^{40}$.

General anesthesia suppresses neuronal signal conduction at various levels of sensory pathways, including olfaction. However, these effects are known to be transient and completely reversible ${ }^{3,21}$. A study by Kostopanagiotou et al..$^{29}$ found that Sevoflourane, administered during general anesthesia, has transient effects on olfactory memory, and that this effect lasted up to $3 \mathrm{~h}$ after surgery. However, the interval for testing may have been too far apart (minimum of three days) to have measured any transient anesthesia effects.

Age was negatively correlated with clinically significant change in threshold score. This means the lower the age, the more likely for threshold scores to improve post-operatively. This was observed particularly more for women than men in this study. Cognitive function may play a role in this finding as retest effects were found to decline with age ${ }^{41}$. Younger participants may have benefitted from retest effects more. Conversely, another 
possible explanation is that older individuals may be more likely to experience cognitive decline. A study by Yahiaoui-Doktor et $\mathrm{al}^{42}$ found an association between worsening of olfactory and cognitive performance.

Women have been found to have better olfactory performance, although the difference between sexes may be small and more observable in larger samples ${ }^{35}$. In our population, men had higher mean scores than women overall; but the significant difference was only observed in threshold scores. A meta-analysis by Sorokowski et $\mathrm{al}^{43}$ found that women outperform men on threshold testing, but only by a small margin. Our comparably small sample size may be the reason why we did not observe a similar trend in our study. However, consistent with our findings, Sorokowski et al. also found that the effect size on threshold was twice as high as that of identification when it comes to differences in sex. Thus, threshold tests may be the most appropriate measure to check for potential differences in olfactory function among sexes ${ }^{43}$.

Sleep deprivation has been found to affect cognitive performance on a range of tasks ${ }^{44-46}$. Our findings were consistent with these as a significant decrease in pre- and post-operative threshold scores and pre-operative TDI scores were noted in patients with self-rated sleep deficits. Although discrimination and identification scores may be more associated with cognition, this may be in support of the idea that inadequate sleep can be associated with poor performance, especially in tasks that require attention and memory. Unfortunately, the amount of sleep deficit was not quantified in this study, as patients were only asked if they felt they have slept sufficiently long or otherwise. A more finite period of sleep deficit may be explored for future research.

Limitations to this study include the following: limited variability in the types of surgeries and anesthesia, low sample size, less than ideal sites of testing, and variable olfactory testing interval. Most were trauma surgeries, and limited information was gathered from other types of surgeries mentioned in literature such as cardiac and abdominal/gastrointestinal surgeries. Majority of our population received general anesthesia as well, and this may have led to biased results. As post-operative change in olfaction among non-nasal surgeries is an outcome that is relatively rarely reported in literature, a larger sample size may have better reflected the true presence or absence of this phenomenon. Although we attempted to best control the setting where the olfactory tests were done, it is unknown to what degree this had an effect to the patients' scores.

Lastly, because this study was done before the pandemic, COVID-19 testing was not mandatory and COVID19 associated olfactory loss was not considered as a variable in this study. However, possible effects of anesthesia on the sense of smell might be more pronounced in patients with pre-existing olfactory loss. Such situations can be expected to be slightly more frequent in the future as a consequence of the COVID-19 pandemic, with its ensuing olfactory loss ${ }^{47}$. Future studies may look at a larger sample, including patients with history of previous olfactory dysfunction (possibly those with previous COVID-19 infection), with standardized olfactory testing intervals to control for retest effects.

\section{Conclusion}

Post-operative olfactory scores remain stable. However, identification scores exhibited a slight increase which can be attributed to a retest effect. Age was negatively correlated with change in threshold score, consistent with retest effects declining with age, as well as the association of olfactory and cognitive dysfunction with aging. Type of surgery or anesthesia had little or no effect on olfactory function. Overall, the present results indicate that surgery outside of the nasal and paranasal sinus region performed in general anesthesia has no major effect on the sense of smell.

Received: 8 September 2021; Accepted: 2 December 2021

Published online: 13 December 2021

\section{References}

1. Hummel, T. et al. Position paper on olfactory dysfunction. Rhinology 54, 1-30 (2017).

2. Landis, B. N., Konnerth, C. G. \& Hummel, T. A study on the frequency of olfactory dysfunction. Laryngoscope 114, 1764-1769 (2004).

3. Kaydu, A., Kiliç, E., Gökçek, E. \& Kaçar, C. Anosmia after caudal epidural steroid injection. Anesth. Essays Res. 12, 291 (2018).

4. Marin, C. et al. Olfactory dysfunction in neurodegenerative diseases. Curr. Allergy Asthma Rep. 18, 42 (2018).

5. Leopold, D. Distortion of olfactory perception: Diagnosis and treatment. Chem. Senses 27, 611-615 (2002).

6. Seiden, A. M. \& Duncan, H. J. The diagnosis of a conductive olfactory loss. Laryngoscope 111, 9-14 (2001).

7. Pade, J. \& Hummel, T. Olfactory function following nasal surgery. Laryngoscope 118, 1260-1264 (2008).

8. Elbistanli, M. S. et al. Significance of medial osteotomy on the olfactory function in patients who underwent septorhinoplasty. J. Craniofac. Surg. 30, E106-E109 (2019).

9. Akkoca, Ö. et al. Comparison of the effects of 2 surgical techniques used in the treatment of concha Bullosa on olfactory functions. Ear Nose Throat J. 99, 437-441 (2020).

10. Lilja, M. et al. Olfactory and gustatory functions after free flap reconstruction and radiotherapy for oral and pharyngeal cancer: a prospective follow-up study. Eur. Arch. Oto-Rhino-Laryngol. 275, 959-966 (2018).

11. Altundag, A., Salihoglu, M., Cayonu, M. \& Tekeli, H. Clinical assessment of olfactory functions in children who underwent adenotonsillectomy during pre- and post-operative period. Int. J. Pediatr. Otorhinolaryngol. 78, 1138-1142 (2014).

12. Fornazieri, M. A. et al. The effects of adenoidectomy on the smell perception of children. Int. Forum Allergy Rhinol. 9, 87-92 (2019).

13. Ung, T. H. et al. Preservation of olfaction in anterior midline skull base meningiomas: a comprehensive approach. Acta Neurochir. (Wien) 161, 729-735 (2019).

14. Shetty, S. R. et al. Limitations of the endonasal endoscopic approach in treating olfactory groove meningiomas. A systematic review. Acta Neurochir. 159, 1875-1885 (2017).

15. Podlesek, D. et al. Olfactory bulb volume changes associated with trans-sphenoidal pituitary surgery. PLoS ONE 14, e0224594 (2019).

16. Lee, J. J. et al. Risk factors for patient-reported olfactory dysfunction after endoscopic transsphenoidal hypophysectomy. JAMA Otolaryngol. Head Neck Surg. 146, 621-629 (2020).

17. Hendrix, P. et al. Perioperative olfactory dysfunction in patients with meningiomas of the anteromedial skull base. Clin. Anat. 32, 524-533 (2019). 
18. Erdem, K. et al. Evaluation of olfactory memory after coronary artery bypass grafting. Kardiochirurgia i Torakochirurgia Pol. 11, 381-384 (2014).

19. Erdem, K. et al. Effects of coronary artery bypass grafting surgery on olfactory and taste functions. Heart Surg. Forum 22, E416E422 (2019).

20. Harris, A. M. \& Griffin, S. M. Postoperative taste and smell deficit after upper gastrointestinal cancer surgery: An unreported complication. J. Surg. Oncol. 82, 147-150 (2003).

21. Dhanani, N. M. \& Jiang, Y. Anosmia and hypogeusia as a complication of general anesthesia. J. Clin. Anesth. 24, 231-233 (2012).

22. Baker, J. J., Öberg, S. \& Rosenberg, J. Loss of smell and taste after general anesthesia. A A Case Rep. 9, 346-348 (2017).

23. Cooper, G. M. An unexpected benefit of anaesthesia. Anaesthesia 53, 830 (1998).

24. Adelman, B. T. Altered taste and smell after anesthesia: Cause and effect?. Anesthesiology 83, 647-648 (1995).

25. Du, W., Xu, Z., Wang, W. \& Liu, Z. A case of anosmia and hypogeusia as a complication of propofol. J. Anesth. 32, 293-296 (2018).

26. Konstantinidis, I., Tsakiropoulou, E., Iakovou, I., Douvantzi, A. \& Metaxas, S. Anosmia after general anaesthesia: A case report. Anaesthesia 64, 1367-1370 (2009).

27. Henkin, R. Reply: Altered taste and smell after anesthesia: cause and effect?. Anesthesiology 83, 648-649 (1995).

28. Elterman, K. G., Mallampati, S. R., Kaye, A. D. \& Urman, R. D. Postoperative alterations in taste and smell. Anesthesiol. Pain Med. 4, 1-4 (2014).

29. Kostopanagiotou, G. et al. Sevoflurane impairs post-operative olfactory memory but preserves olfactory function. Eur. J. Anaesthesiol. 28, 63-68 (2011).

30. Sari, H. et al. Short term olfactory memory and olfactory function after inhalation anesthetic agents: a randomized clinical trial. Brazilian J. Anesthesiol. 70, 36-41 (2020).

31. Cassidy, B. \& McCoy, D. G. L. The sweet smell of success. Anaesthesia 55, 823-823 (2001).

32. Salvinelli, F., Casale, M., Hardy, J. F., D’Ascanio, L. \& Agrò, F. Permanent anosmia after topical nasal anaesthesia with lidocaine 4\%. Br. J. Anaesth. 95, 838-839 (2005).

33. Welge-Lüssen, A., Wille, C., Renner, B. \& Kobal, G. Anesthesia affects olfaction and chemosensory event-related potentials. Clin. Neurophysiol. 115, 1384-1391 (2004).

34. Hummel, T., Hummel, C. \& Welge-Luessen, A. Assessment of Olfaction and Gustation. in Management of Smell and Taste Disorders: A Practical Guide for Clinicians 58-75 (Thieme, 2013).

35. Oleszkiewicz, A., Schriever, V. A., Croy, I., Hähner, A. \& Hummel, T. Updated Sniffin' Sticks normative data based on an extended sample of 9139 subjects. Eur. Arch. Oto-Rhino-Laryngol. 276, 719-728 (2019).

36. Gudziol, V., Lötsch, J., Hähner, A., Zahnert, T. \& Hummel, T. Clinical significance of results from olfactory testing. Laryngoscope 116, 1858-1863 (2006)

37. Scharfen, J., Peters, J. M. \& Holling, H. Retest effects in cognitive ability tests: A meta-analysis. Intelligence 67, 44-66 (2018).

38. Lievens, F., Reeve, C. L. \& Heggestad, E. D. An examination of psychometric bias due to retesting on cognitive ability tests in selection settings. J. Appl. Psychol. 92, 1672-1682 (2007).

39. Hedner, M., Larsson, M., Arnold, N., Zucco, G. M. \& Hummel, T. Cognitive factors in odor detection, odor discrimination, and odor identification tasks. J. Clin. Exp. Neuropsychol. 32, 1062-1067 (2010).

40. Hummel, T., Sekinger, B., Wolf, S. R., Pauli, E. \& Kobal, G. 'Sniffin' sticks'. Olfactory performance assessed by the combined testing of odor identification, odor discrimination and olfactory threshold. Chem. Senses 22, 39-52 (1997).

41. Scharfen, J., Jansen, K. \& Holling, H. Retest effects in working memory capacity tests: A meta-analysis. Psychon. Bull. Rev. 25, 2175-2199 (2018).

42. Yahiaoui-Doktor, M. et al. Olfactory function is associated with cognitive performance: results from the population-based LIFEAdult-Study. Alzheimers Res. Ther. 11(1), 43. https://doi.org/10.1186/s13195-019-0494-z (2019).

43. Sorokowski, P. et al. Sex differences in human olfaction: A meta-analysis. Front. Psychol. 10, 1-9 (2019).

44. Lim, J. \& Dinges, D. A meta-analysis of the impact of short-term sleep deprivation on cognitive variables. Psychol. Bull. 136, 375-389 (2010).

45. Mantua, J. \& Simonelli, G. Sleep duration and cognition: is there an ideal amount?. Sleep 42, 1-3 (2019).

46. Holding, B. C., Ingre, M., Petrovic, P., Sundelin, T. \& Axelsson, J. Quantifying cognitive impairment after sleep deprivation at different times of day: A proof of concept using ultra-short smartphone-based tests. Front. Behav. Neurosci. 15, 666146 (2021).

47. Brann, D. H. et al. Non-neuronal expression of SARS-CoV-2 entry genes in the olfactory system suggests mechanisms underlying COVID-19-associated anosmia. Sci. Adv. 6, 1-20 (2020).

\section{Acknowledgements}

We would like to thank Prof. Hans Zwipp for his very helpful comments at the initiation of the study.

\section{Author contributions}

A.K.H.: Conceptualization, Formal analysis, Writing—review and editing. P.F.: Conceptualization, Data collection, Writing - review and editing. A.H.: Conceptualization, Supervision, Writing-review and editing. T.H.: Conceptualization, Formal analysis, Supervision, Writing_-review and editing.

\section{Funding}

Open Access funding enabled and organized by Projekt DEAL.

\section{Competing interests}

The authors declare no competing interests.

\section{Additional information}

Correspondence and requests for materials should be addressed to A.K.H.

Reprints and permissions information is available at www.nature.com/reprints.

Publisher's note Springer Nature remains neutral with regard to jurisdictional claims in published maps and institutional affiliations. 
(c) (i) Open Access This article is licensed under a Creative Commons Attribution 4.0 International cc) License, which permits use, sharing, adaptation, distribution and reproduction in any medium or format, as long as you give appropriate credit to the original author(s) and the source, provide a link to the Creative Commons licence, and indicate if changes were made. The images or other third party material in this article are included in the article's Creative Commons licence, unless indicated otherwise in a credit line to the material. If material is not included in the article's Creative Commons licence and your intended use is not permitted by statutory regulation or exceeds the permitted use, you will need to obtain permission directly from the copyright holder. To view a copy of this licence, visit http://creativecommons.org/licenses/by/4.0/.

(C) The Author(s) 2021 